\title{
SUBMARINE POSITION INFORMATION FOR ACOUSTIC MEASUREMENTS
}

\author{
B. R. Bjork \\ Naval Surface Warfare Center \\ Carderock Division, Detachment Puget Sound \\ Bremerton, WA 98314-5215
}

\author{
W. F. Fagan \\ Naval Surface Warfare Center \\ Carderock Division, Detachment Puget Sound \\ Bremerton, WA 98314-5215
}

\begin{abstract}
Conducting noise measurements of US submarines involves maneuvering the ship near acoustic measurement arrays. Real time position information relative to the arrays is required to ensure the safety of both submarine and the arrays. Position information is also required to correct acoustic data for variations in distance to the arrays that may occur over the course of the testing. This paper describes the tracking system employed by the Southeast Alaska Acoustic Measurement Facility (SEAFAC) which is the U.S. Navy's newest acoustic measurement facility.
\end{abstract}

\section{INTRODUCTION}

Acoustic signature measurements of U.S. submarines are acquired by maneuvering the ship, between two submerged acoustic measurement arrays spaced about 250 yards apart (Fig. 1). This close proximity to the arrays is necessary to ensure a sufficiently high signal to noise ratio.

Accurate position information is necessary during acoustic measurements for two reasons. First, we must minimize exposure of the submarine to hazards when maneuvering close to range boundaries on arrays. Secondly, we must correct the measured data to account for the distance between the submarine and the arrays.

The Southeast Alaska Acoustic Measurement Facility (SEAFAC), located in Behm Canal near Ketchikan, Alaska (Fig. 2), employs a tracking system designed by engineers at the Naval Surface Warfare Center Carderock Division, Detachment Puget Sound in conjunction with hardware built by the University of Washington Applied Physics Laboratory (UW/APL) and software developed by Offshore Systems Limited (OSL) of Canada. The tracking system called SEAFAC Tracking and Navigation Aids Segment (STANAS) satisfies the two conditions requiring accurate position information.

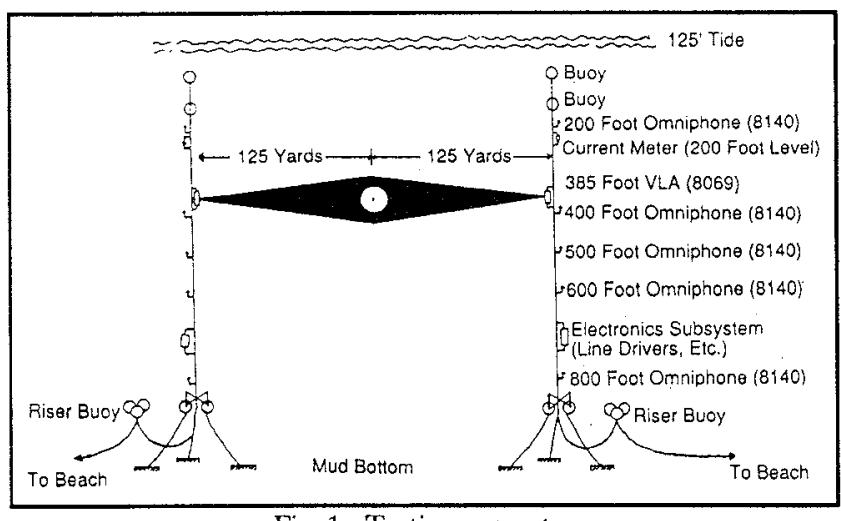

Fig. 1. Testing geometry.

STANAS provides real time, precise 3-D position information to the submarine while operating within SEAFAC's 5,000 yds by 12,000 yds underway operating area. The information is provided to the ship via an acoustic data link on a continuous basis. STANAS also tracks the acoustic measurement arrays and updates their position due to tidal drift. This information in addition to the submarine location, is provided to the SEAFAC data acquisition system.

SEAFAC is located in inland waters which pose hazards to the submarine should it deviate outside the underway operating area (Fig. 3). To ensure ship safety, the STANAS includes an Underwater Emergency Warning System (UEWS) which alerts the submarine that it is straying out of the operating area. The UEWS emits a series of tones which the ship's SONAR and other acoustic sensors will detect. The tracking system in-water components consist of eight bottom mounted arrays, two underwater communications transducers, and two emergency acoustic warning projectors. 


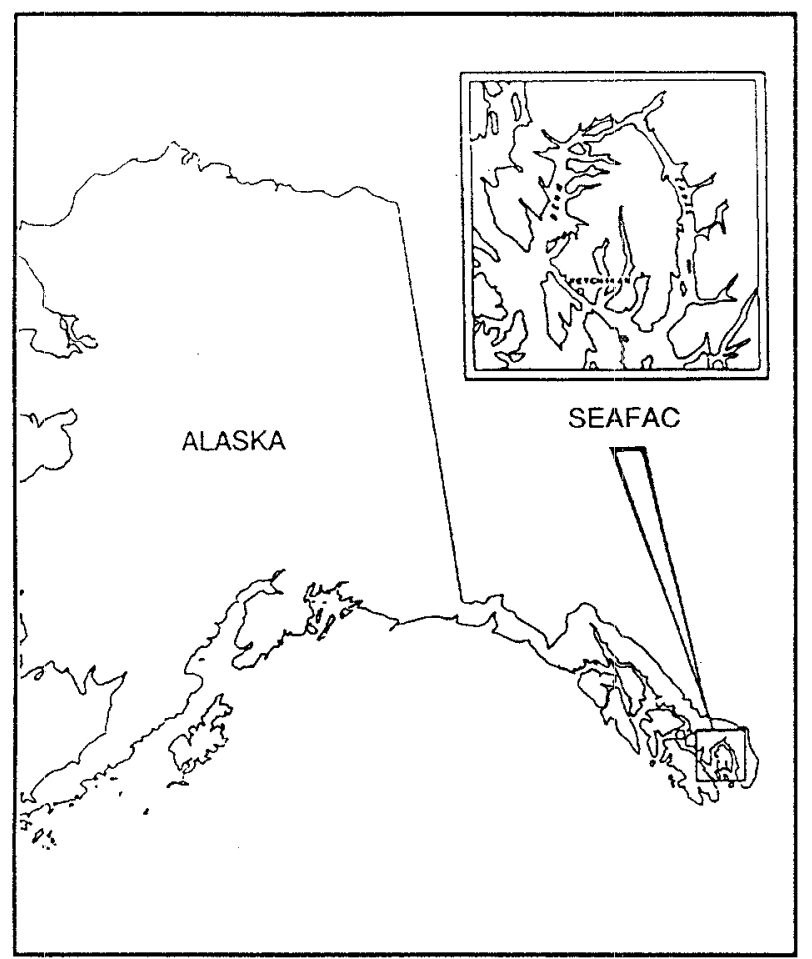

Fig. 2. SEAFAC location.

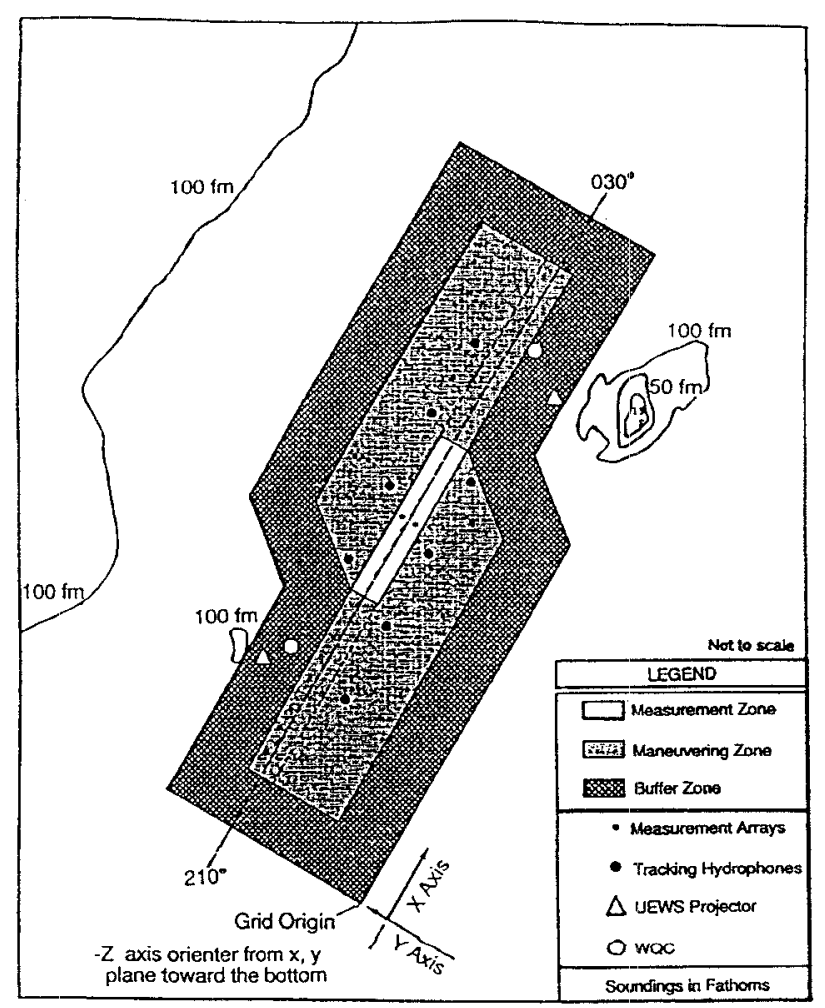

Fig. 3. SEAFAC operating area layout.

\section{OPERATING AREA PHYSICAL DESCRIPTION}

\section{A. Operating Area Size}

The size of the operating area of SEAFAC is dependent on two variables. The first is the characteristics of the measurement arrays and the second is the maneuverability of the ship.

A measurement array spacing of 250 yards balances the constraints of the tracking system while overcoming the effects of ambient noise on acoustic measurements. U.S. acoustic engineering measurement facilities similar to SEAFAC have used spacings between 230 and 260 yards. The experience gained at these facilities along with the improved sensors used at SEAFAC ensures that a distance of 250 yards provides for safe navigation of the submarine. It also guarantees a sufficient acoustic signal to noise ratio of the submarine signature over ambient noise to allow for valid data acquisition.

Submarine acoustic measurements are taken on the bow, beam, and stern aspects of the ship (Fig. 4) while transiting through the midpoint space between the acoustic measurement arrays. Bow and stern data are collected between 800 and 1,500 yards away from the arrays, resulting in a data collection zone of nominally 3,000 yards centered at the arrays.

In addition to the 3,000 yard data collection zone, maneuvering room is provided for the submarine to conduct turns, regain a steady course, and prepare to pass through the arrays on the reverse course line. The distance provided for this is 3,500 yards at each end of the data collection zone. Thus overall length of 10,000 yards and a width of 3,500 yards is required for standard operations. An additional 1,000 yard buffer zone is added to all sides of the operating area of SEAFAC to further ensure safe navigation. Therefore, the total operating area covers 12,000 yards by 5,000 yards (Fig. 5 ).

\section{B. Behm Canal}

Behm Canal, located about 10 miles north of Ketchikan Alaska, proved to be an excellent choice for SEAFAC based on acoustics. However, unique topography characteristics of the area resulted in several special considerations for the STANAS. A major consideration for safe tracking is the location of the two sea mounts in the general area. The SEAFAC underway operating area is oriented along the axis of $030^{\circ} \mathrm{T}$ and $210^{\circ} \mathrm{T}$ to significantly reduce exposure to these sea mounts. 


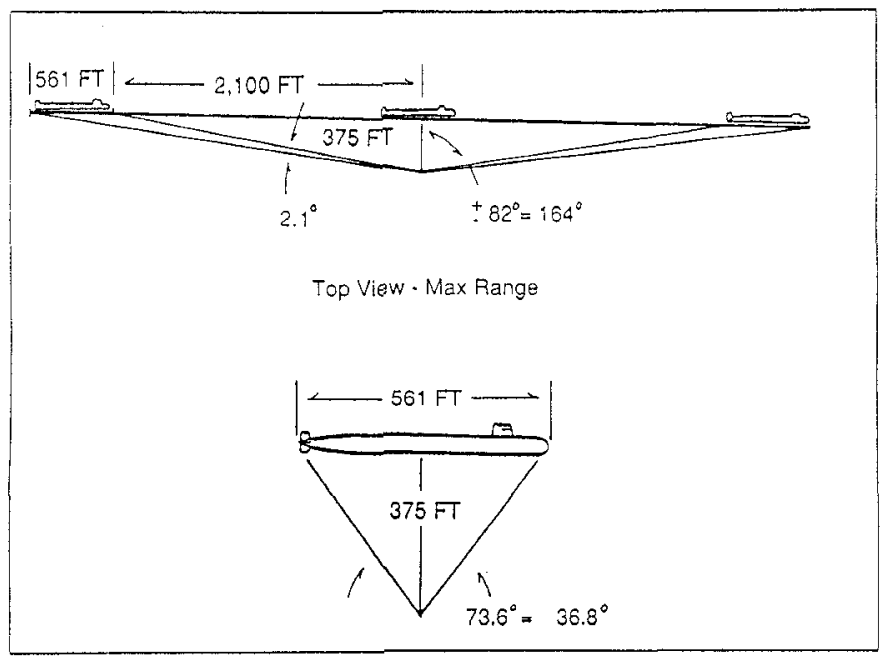

Fig. 4. Data acquisition geometry.

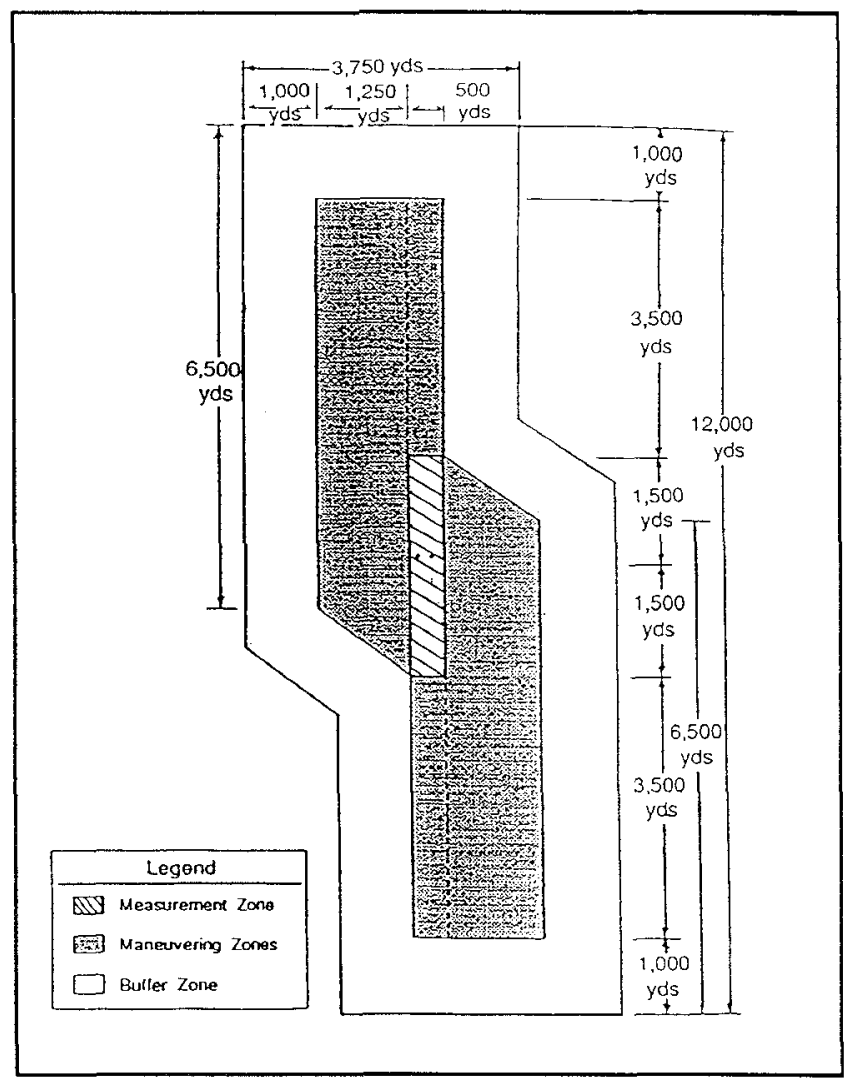

Fig. 5. SEAFAC dimensions.

Another constraint of the topography is the irregularity of the sea bed over the length of the SEAFAC underway operating area. Figs. 6, 7, and 8 present the varying topography with views of the north, central, and southern cross sections of the underway operating area. Exact placement of the tracking hydrophones is essential to ensure that they will not be shadowed by the surrounding topography. In addition, hydrophone location and depth is entered into the tracking algorithm to correct for varying arrival times of the signal from the tracking pinger.

\section{TECHNICAL REQUIREMENTS}

The topography of SEAFAC plus the ship's maneuverability characteristics determine the location and number of ocean bottom mounted tracking arrays employed. The tracking system is required to provide accurate position information over the entire operating area, and to transmit the data in real time to the submarine so it can maneuver safely.

Overall technical requirements for the STANAS system and its component subsystems are derived from general requirements specified in the Naval Operating Instructions. These instructions define the minimum safety requirements for the conduct of submerged submarine operations in restricted waters on instrumented ranges. They impose the following general regulations:

The ship must be tracked at all speeds and depths.

Sufficient navigation information must be provided to the ship to ensure that it can maneuver safely.

All communications dealing with navigation positions must be recorded.

A means of warning the ship must be available should the ship deviate from expected course or during a possible loss of underwater communications (WQC).

Surface vessels in the vicinity of the operating area must be tracked by radar.

A record of tracked vehicle positions must be stored.

To ensure that these requirements were met, the tracking system at SEAFAC was built to meet the following specifications:

A submarine can be tracked over the entire SEAFAC operating area (measurement, maneuvering, and buffer zones.)

The system can track up to 6 targets at any one time. Generally, during an acoustic trial, there are only four targets to track (the submarine, one surface vessel, and the two measurement arrays). 


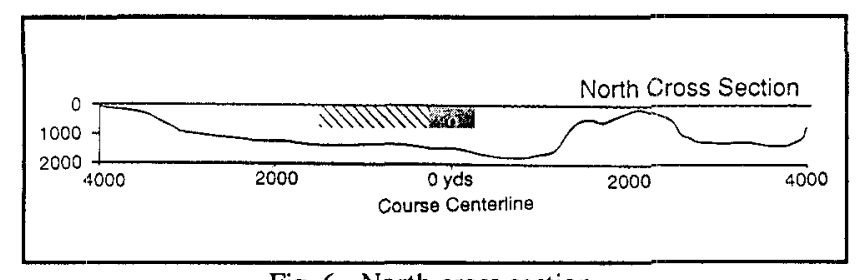

Fig. 6. North cross section.

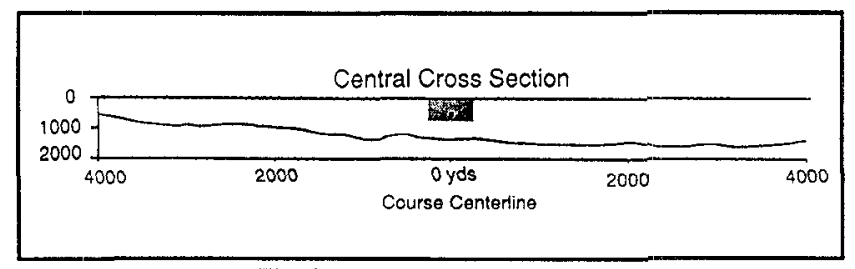

Fig. 7. Central cross section.

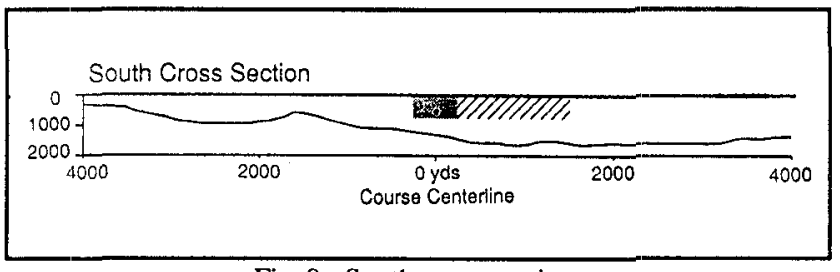

Fig. 8. South cross section.

Visual navigation aids are placed around the operating area to allow the submarine to verify tracking system performance with standard navigational fixes.

Position certainty (fix quality) and clock synchronization are calculated continuously.

An Underwater Emergency Warning System (UEWS) projector is on each end of the operating area to warn the submarine that it is crossing into the buffer zone and must take action to re-enter the operating area.

Displays showing both the numerical $(X, Y)$ and geographical positions of the submarine are located in SEAFAC control and on the submarine being tracked.

The accuracy of the tracking system is \pm 1 yard and \pm 0.5 knots for vehicles up to 50 knots in the vicinity of the arrays.

The frequency and pulse rate of the tracking pinger are selected to have minimum interference with the acoustic measurements being performed.
This system is based on existing hardware and was designed to minimize complexity and risk, yet maximize performance.

\section{EQUIPMENT DESCRIPTION}

\section{A. Tracking System Overview}

STANAS is a 3 dimensional synchronous tracking system that calculates a ship's position in the underway operating area with an accuracy of \pm 1 yard near the center of the area and \pm 5 yards at the extremes. Asynchronous tracking is also available.

The system consists of two highly stable synchronized clocks. One clock is placed on board the submarine to control the pinger pulse rate, and the other in the shore based laboratory facility to maintain a precisely synchronized signal between the two locations. At eight second intervals the synchronization clock on board the submarine triggers a $16 \mathrm{kHz}$ pulse emitted by a pinger externally mounted to the hull of the ship. During measurement acquisition the emitted pulse is switched to $75 \mathrm{kHz}$ to reduce contamination with the ship signature. The pulse is Phase Shift Keyed (PSK) with a 20 bit correlation code and 28 bits of data.

The tracking pulse is received at eight tracking arrays located along the operating area course at an average depth of 1,300 feet. The received signal is amplified and cabled to the shore installation for processing. With matching correlation code receivers ( 8 channels at $75 \mathrm{kHz}$ and 8 channels at $16 \mathrm{kHz}$ ) the effects of shipping noise, spurious biologic noise, and multipath signals are essentially eliminated from processed returns.

The processed position data are displayed in the laboratory and then transmitted to the submarine acoustically as an encoded message from one of the eight tracking arrays (acoustic data link). The data link is received onboard the submarine via the pinger and decoded for navigation display. Additionally, automated synthesized voice position information is available via WQC should problems be encountered with the data link.

\section{B. Tracking Arrays}

Each tracking array contains a projector and a hydrophone. The projector is used for emitting the data link. The hydrophone receives the pulse from the submarine. The projector is resonant at the $16 \mathrm{kHz}$ data link frequency. The hydrophone has wide band characteristics operating from $8 \mathrm{kHz}$ to $75 \mathrm{kHz}$. A hydrophone preamplifier boosts 


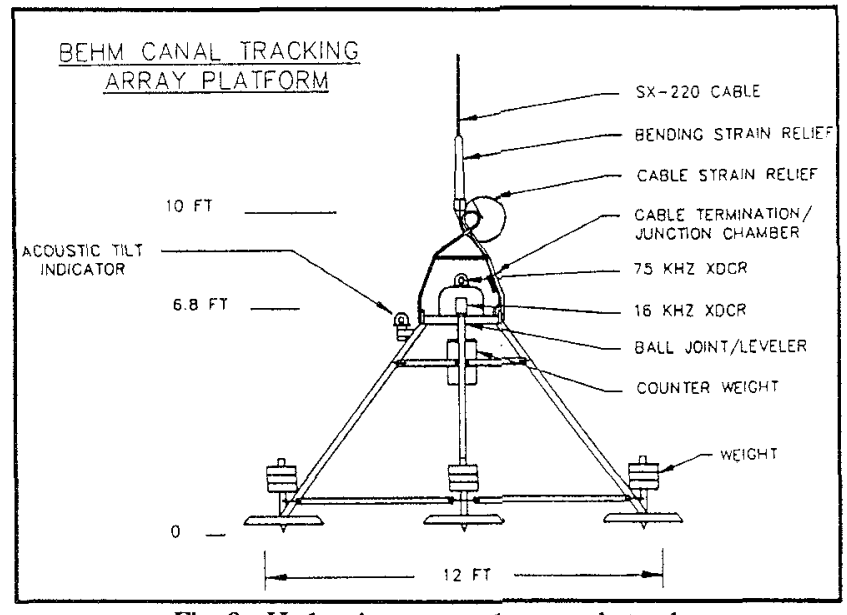

Fig. 9. Hydrophone transducer and stand.

the received signal for transmission and also contains a circuit to protect the preamplifier from damage during data transmissions from the projector. The array mooring structure includes a ball joint leveler that provides a level platform for the transducer on the uneven seabed (Fig. 9).

\section{WQC Transducers and Underwater Emergency Warning Projectors}

The two WQC assemblies are similar to the eight tracking arrays. Each one contains a hydrophone and a projector sharing a common coaxial signal cable. The projector is a commercially available unit. The receive hydrophone has band limited characteristics for the WQC high band $(8 \mathrm{kHz}$ to $11 \mathrm{khz}$ ) and also contains a preamplifier to compensate for cable attenuation loss.

Each projector of the two UEWS are wide band projectors which emit a series of tones designed to be detected on the submarines tactical SONAR as well as the WQC.

\section{Cabling}

The signal cables returning to shore are each over five miles long. The coaxial cable used is known as AUTEC cable and has the SIMPLEX cable designation SX220.

\section{E. Shore Based Acoustic Receiver/Transmitter}

The Shore Based Acoustic Receiver/Transmitter System (SARTS) is located at the range operations building. The eight cables from the tracking arrays connect to the ARTS which contains the PSK and leading edge detection receivers. The leading edge detection receivers are doppler compensated to minimize the detection of false signals. The underwater position of the submarine or other tracked objects is determined by measuring the travel times of an acoustic pulse emitted by the tracked object and received by bottom mounted tracking hydrophones. The travel time measurement, coupled with known sound velocity along the acoustic path permits the computation of the position of the tracked object relative to the bottom mounted tracking hydrophones. The ARTS determines pulse arrival time and sends the timing data to the tracking computer for processing and display of vessel track. The coordinates of the tracked object are returned to the submarine via the acoustic data link. The tracking computer selects the tracking array most appropriate for transmission to the submarine and controls the output level transmitted. The position of the submarine is continuously monitored as well as the quality of the tracking solution. In the event that tracking is lost or the ship crosses into the buffer area, the UEWS signal is enabled by the ARTS. The warning signal can also be activated manually at any time by the tracking system operator or the Trial Director.

\section{F. Ship Based Acoustic Receiver/Transmitter}

STANAS equipment is placed on the submarine for each acoustic trial. This equipment consists of an externally mounted pinger and the Ship Based Acoustic Receiver/ Transmitter (SARTS) which includes a data processing computer, video display, and a duplex interface between the pinger and the computer. The computer controls the ping rate, frequency, and power of the ship's pinger. It also decodes the data link information and plots the ship position on the SARTS video display every 8 seconds.

\section{SUMMARY}

The Southeast Alaska Acoustic Measurement Facility (SEAFAC) employs the SEAFAC Tracking and Navigation Aids System (STANAS) to track submarines during their acoustic measurements.

STANAS components are "off the shelf" technology. STANAS performs the functions of acquiring tracking information and processing the data. It then transmits the information to the submarine and the acoustic measurement data acquisition systems for use in positioning the ship and range correcting the acoustic data respectively.

STANAS solves the challenges posed by the ambient conditions of the operating area by using synchronized signals and phase shift keyed modulation pulses between the submarine and the shore facility.

Presently STANAS has successfully tracked submarines for over 170 hours of acoustic measurements. Its performance is reliable and predictable. 